\title{
Reconstructing Binary Matrices with Neighborhood Constraints: An NP-hard Problem
}

\author{
A. Frosini ${ }^{1}$, C. Picouleau ${ }^{2}$, and S. Rinaldi ${ }^{3}$ \\ ${ }^{1}$ Dipartimento di Sistemi e Informatica \\ Università di Firenze, (Firenze, Italy) \\ andrea.frosini@unifi.it \\ ${ }^{2}$ Laboratoire CEDRIC CNAM, \\ (Paris, France) \\ ${ }^{3}$ Dipartimento di Scienze Matematiche ed Informatiche \\ Università di Siena, (Siena, Italy)
}

\begin{abstract}
This paper deals with the reconstruction of binary matrices having exactly $-1-4-$ adjacency constraints from the horizontal and vertical projections. Such a problem is shown to be non polynomial by means of a reduction which involves the classic NP-complete problem 3color. The result is reached by bijectively mapping all the four different cells involved in 3-color into maximal configurations of 0 s and 1s which show the adjacency constraint, and which can be merged into a single binary matrix.
\end{abstract}

Keywords: Discrete Tomography, polynomial time reduction, NPcomplete Problem.

\section{Introduction and Notations}

Given a binary matrix, its horizontal and vertical projections are defined as the sum of its elements for each row and each column, respectively. The reconstruction of a binary matrix from its orthogonal projections has been studied by Ryser [78. One can refer to the books of Herman and Kuba 415] for further information on the theory, algorithms and applications of such a kind of classical problems which fit in the wide area of the discrete tomography.

It is well-known that this basic problem, where the only constraints to verify are both projections, can be solved in polynomial time. Numerous studies deal with the same problem when additional local or global constraints are imposed. Here we consider binary matrices with a local adjacency constraint, and we show a polynomial time reduction which maps each instance of the classical NPcomplete problem 3-color into an instance of their consistency problem. Then we show that such a reduction fulfills a series of requirements stated in a general framework, in 2, and allows us to set the time complexity of 3-color as lower bound to that of our problem.

Now, let us proceed by introducing basic notations and definitions: given a $m \times n$ binary matrix $M$ we denote by $M[i, j]$ the element in position $(i, j)$. The 
horizontal projection of $M$ is defined as the vector $H=\left(h_{1}, \ldots, h_{m}\right)$ such that for each $1 \leq i \leq m, h_{i}$ counts the number of 1's on row $i$. Similarly, the vertical projection $V=\left(v_{1}, \ldots, v_{n}\right)$ of $M$, is the vector such that for each $1 \leq j \leq n, v_{j}$ counts the number of 1 's on column $j$.

In order to define the adjacency constraint, we rely on the definition of the neighborhood of a given internal element $M[i, j], 1<i<m, 1<j<n$, of the binary matrix $M$ : we speak of 4 -adjacency if its neighbors are considered to be the elements $M[i, j-1], M[i, j+1], M[i-1, j]$ and $M[i+1, j]$ i.e. the horizontal and vertical adjacent cells. Different kinds of adjacency can be defined as well, but they do not concern this paper.

We say that the matrix $M$ fulfills the exactly-1-4-adjacency constraint if $M[i, j]=1$ implies that there is exactly one among its 4-adjacent cells that has value 1 . The class of binary matrices that fulfill exactly-1-4-adjacency is denoted by $\mathcal{N}_{4}^{=1}$.

The concept of adjacent constraint has a prominent role when dealing with scheduling problems (see [6]), and it has been recently studied under a tomographical perspective in [1].

Here, two classical problems related to the class $\mathcal{N}_{4}^{=1}$ are addressed:

$\operatorname{Consistency}\left(\mathcal{N}_{4}^{=1}, H, V\right)$

Input: a couple of integer vectors $H$ and $V$;

Question: does there exist an element of the class $\mathcal{N}_{4}^{=1}$ whose horizontal and vertical projections are the vectors $H$ and $V$, respectively?

Reconstruction $\left(\mathcal{N}_{4}^{=1}, H, V\right)$

Input: a couple of integer vectors $H$ and $V$;

Task: reconstruct an element of the class $\mathcal{N}_{4}^{=1}$ whose horizontal and vertical projections are the vectors $H$ and $V$, respectively, if it exists, otherwise give a failure.

The condition $\sum_{i=1}^{m} h_{i}=\sum_{j=1}^{n} v_{j}$ is obviously necessary for the existence of a binary matrix respecting both projections in the two problems.

Finally, we define the class of colored matrices and their projections: given a set of colors $C=\left\{c_{1}, \ldots, c_{k}\right\}$, a $k$-colored $m \times n$ matrix $A$ is a matrix whose elements are in $C \cup\{$ colorless $\}$.

The projection of the $i$ th row of $A$ is the $k$ dimensional vector $\left(h_{i}^{c_{1}}, h_{i}^{c_{2}}, \ldots\right.$, $h_{i}^{c_{k}}$ ), where each coordinate $h_{i}^{c_{s}}$ counts the number of elements of color $c_{s} \in C$ lying in the $i$ th row. A similar definition of vertical projection can be given for a generic column $j$ of $A$ (see Fig. 1). The sequence of all the horizontal [resp. vertical] projections is indicated as $H k$ [resp. $V k]$.

\section{Principle of the Reduction}

In order to prove our main result, i.e. the NP-completeness of Consistency $\left(\mathcal{N}_{4}^{=1}, H, V\right)$, we consider the following classical problem: 
$(1,0,1) \quad(1,0,2)$

$(0,2,1):(0,0,0):(0,1,1)$

\begin{tabular}{|l|l|l|l|l|}
\hline$b$ & & & $y$ & \\
\hline$r$ & $y$ & & $r$ & $r$ \\
\hline$b$ & $r$ & & $r$ & $b$ \\
\hline
\end{tabular}

Fig. 1. A 3-color matrix with the colors $C=\{\mathbf{y} e l l o w, \mathbf{b} l u e, \mathbf{r e d}\}$, and its projections

$3-$ color:

let $C=($ yellow, blue, red) be a set of three colors.

Input: two 3 dimensional integer vectors

$$
H 3=\left(\left(h_{1}^{y}, h_{1}^{b}, h_{1}^{r}\right), \ldots,\left(h_{m}^{y}, h_{m}^{b}, h_{m}^{r}\right)\right) \text { and } V 3=\left(\left(v_{1}^{y}, v_{1}^{b}, v_{1}^{r}\right), \ldots,\left(v_{n}^{y}, v_{n}^{b}, v_{n}^{r}\right)\right) .
$$

Question: does there exists a 3-color matrix of dimension $m \times n$ whose horizontal and vertical projections are the vectors $H 3$ and $V 3$, respectively?

As for the class $\mathcal{N}_{4}^{=1}$, we can define the correspondent 3-color reconstruction problem.

Inside the 3-color matrix, we refer to each color in $C$ using its initial letter, and to the colorless element using the symbol $c$. Furthermore, it will be useful to introduce the notations

$$
h_{i}^{c}=n-h_{i}^{y}-h_{i}^{b}-h_{i}^{r} \quad \text { and } \quad v_{j}^{c}=m-v_{j}^{y}-v_{j}^{b}-v_{j}^{r}
$$

to denote the number of $c$ elements in a 3-color matrix.

Now, following 2], we define a polynomial time process, say a reduction, from an instance $I$ of 3 -color to an instance $I^{\prime}$ of Consistency $\left(\mathcal{N}_{4}^{=1}, H, V\right)$ by using the correspondence of each color $c, y, b$, and $r$ of $I$ with four different placements of $0 \mathrm{~s}$ and $1 \mathrm{~s}$ in a configuration of cells which satisfy the exactly-1-4-adjacency constraints, as shown in Fig. 2.

The reduction we are going to define has to fulfill two requirements (conditions $n p c 1$ and $n p c 2$ of [2]):

i) from the instance $I^{\prime}$ we can univocally compute back the instance $I$;

ii) each solution of $I^{\prime}$ contains (in a set of fixed positions) only the four chosen configurations.

We achieve $i$ i) by considering the placements of 0 s and 1 s in Fig. 2 which are the only four ones that satisfy the exactly $-1-4-$ adjacency constraint, and that are maximal, i.e. that have the maximum number of $1 \mathrm{~s}$.

Such a reduction sets the computational complexity of 3-color as lower bound to that of Consistency $\left(\mathcal{N}_{4}^{=1}, H, V\right)$. Since the first is known to be NP-complete (see [3]), then the same holds for the latter. 


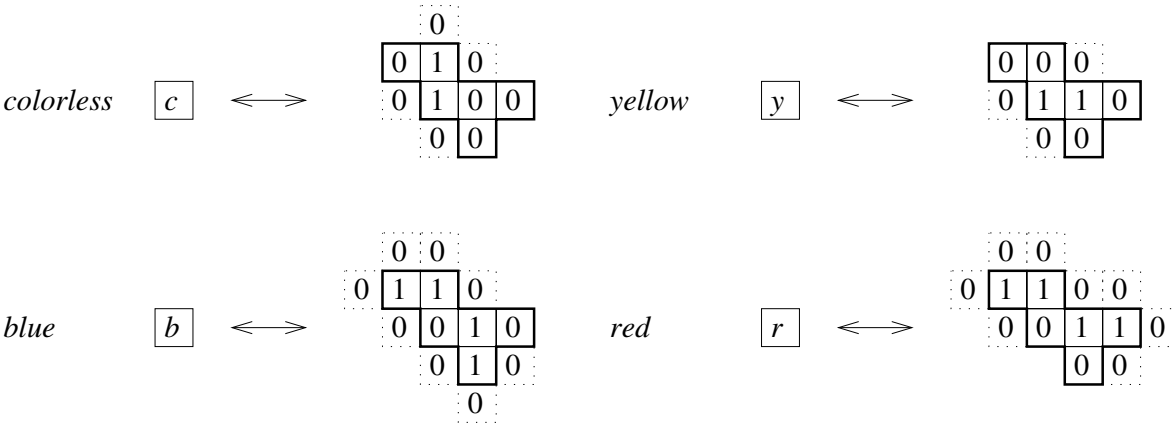

Fig. 2. The correspondence between each atom of the 3 -atom problem and a configuration of elements 0 and 1 having the exactly-1-4-adjacency constraint. The values 0 in the dotted positions do not belong to the chosen configurations, but they are also fixed.

\section{Going Back and Forth from Instance $I$ to Instance $I^{\prime}$}

So, let $I=(H 3, V 3)$ be an instance of 3-atom, with

$$
H 3=\left(\left(h_{1}^{y}, h_{1}^{b}, h_{1}^{r}\right), \ldots,\left(h_{m}^{y}, h_{m}^{b}, h_{m}^{r}\right)\right) \text { and } V 3=\left(\left(v_{1}^{a}, v_{1}^{b}, v_{1}^{r}\right), \ldots,\left(v_{n}^{a}, v_{n}^{b}, v_{n}^{r}\right)\right) .
$$

We compute an instance $I^{\prime}=(H, V)$, with

$$
H=\left(h_{1}, \ldots, h_{6 m+1}\right) \quad \text { and } \quad V=\left(v_{1}, \ldots, v_{12 n}\right)
$$

of Reconstruction $\left(\mathcal{N}_{4}^{=1}, H, V\right)$, as follows: for each $0 \leq i<m$, the entries of the vector $H$ are

$$
\begin{array}{ll}
-h_{6 i+1}=h_{6 m+1}=8 n & \bullet h_{6 i+2}=2 n \\
\text { - } h_{6 i+3}=4 n & \bullet h_{6 i+4}=2 n+h_{i}^{-}+2 h_{i}^{b}+2 h_{i}^{r} \\
\text { - } h_{6 i+5}=3 n+h_{i}^{-}+2 h_{i}^{y}+h_{i}^{b}+2 h_{i}^{r} & \bullet h_{6 i+6}=n+h_{i}^{b}
\end{array}
$$

for each $0 \leq j<n$, the entries of the vector $V$ are

- $v_{12 j+1}=v_{12 j+2}=m+1$

- $v_{12 j+4}=m+1+v_{j}^{b}+v_{j}^{r}$

- $h_{12 j+6}=2 m+v_{j}^{y}+2 v_{j}^{b}+v_{j}^{r}$

- $h_{12 j+8}=2 m+1$

- $h_{12 j+10}=h_{12 j+11}=3 m+1$
- $v_{12 j+3}=4 m$

- $v_{12 j+5}=m+1+2 v_{j}^{-}+v_{j}^{y}+v_{j}^{b}+v_{j}^{r}$

$\bullet h_{12 j+7}=m+1+v_{j}^{r}$

- $h_{12 j+9}=2 m$

- $h_{12 j+12}=0$.

As one can observe, most of the horizontal and vertical projections are independent from the vectors $H 3$ and $V 3$ : they are introduced in order to create the exact shape of the four different configurations used in the reduction.

On the other hand, the projections which involve the entries of the vectors $H 3$ and $V 3$ can be easily understood by referring to the different positions of the $1 \mathrm{~s}$ in the configurations of Fig. 2,

What we have to show is how to go back from instance $I^{\prime}$, obtained from the previous computation, to the correspondent instance $I$. 
This last step fulfills condition $i$ ) given in the previous paragraph, and it implies that the projections of the four chosen configurations are affine linear independent. This check is crucial, since projections which are dependent may mix together, and give rise to solutions of $I^{\prime}$ which do not have a correspondent in the class of the solutions of $I$.

For what concerns the entry $\left(h_{i}^{y}, h_{i}^{b}, h_{i}^{r}\right)$, with $0 \leq i<m$, of the horizontal projections $H 3$ of $I$, its elements can be easily computed by solving the system of equations

$$
\left\{\begin{array}{l}
h_{i}^{c}+2 h_{i}^{b}+2 h_{i}^{r}=h_{6 i+4}-2 n \\
h_{i}^{c}+2 h_{i}^{y}+h_{i}^{b}+2 h_{i}^{r}=h_{6 i+5}-3 n \\
h_{i}^{b}=h_{6 i+6}-n \\
h_{i}^{c}+h_{i}^{y}+h_{i}^{b}+h_{i}^{r}=n
\end{array}\right.
$$

The existence of an integer solution for this system of equations directly follows from the definition of the horizontal projections of the four configurations depicted in Fig. 2 .

A similar system can be defined for the vector $V 3$ of the vertical projections in the instance $I$.

\section{From a Solution of $I$ to a Solution of $I^{\prime}$}

Now we proceed in showing that the instances $I$ and $I^{\prime}$ are equivalent, i.e. that the process of defining instance $I^{\prime}$ from $I$ preserves the existence of its solutions. In the sequel we establish even more (but this is not unusual): there exists a oneto-one correspondence between the set of solutions of $I$ and the set of solutions of $I^{\prime}$.

Intuitively, a solution of $I^{\prime}$ is constructed starting from a solution of $I$, by means of the correspondence in Fig. 2, On the other hand, each solution of $I^{\prime}$ has some fixed positions where one can detect the configurations corresponding to a color, and only those. The horizontal and vertical projections of the instance $I^{\prime}$ defined in the previous paragraph accomplish this task.

So, let us go into details by showing, with the aid of Fig. 3. how to compute a solution $M^{\prime}$ of the instance $I^{\prime}$ from a generic solution $M$ of $I$ : let $1 \leq i \leq m$ and $1 \leq j \leq n$

Step 1: for each element $M[i, j]$, we insert in the matrix $M$ the rectangular configuration of 0 and 1 depicted in Fig. 3, iii), placing its left-uppermost element in position $M[6(i-1)+1,12(i-1)+1]^{\prime}$;

Step 2: according with the color of the element $M[i, j]$, we place a configuration of $0 \mathrm{~s}$ and $1 \mathrm{~s}$ as in Fig. 2 inside the void elements of the corresponding rectangle;

Step 3: we copy the entries of the first row of the matrix $M^{\prime}$ in its last row of index $6 m+1$.

An easy check reveals that the defined matrix $M^{\prime}$ is a solution of instance $I^{\prime}$, as desired. 


\section{From a Solution of $I$ Back to a Solution of $I^{\prime}$}

On the other hand, in order to associate to each solution $M^{\prime}$ of $I^{\prime}$ a solution $M$ of $I$, we need to inspect its entries and detect some of them which are fixed. Figure 3 will help the reader, showing a part of matrix $M$ in detail: let $0 \leq i<m$ and $0 \leq j<n$

i) columns $12 j+12$ are completely filled with 0 s; rows $6 i+1$, and row $6 m+1$ have projections $8 n$, so they are determined by the exactly-1-4-adjacency constraint (see Fig. 3, $i)-i i)$ );

ii) the sets of columns $-12 j+3$ whose projection is $4 m$;

$-12 j+1$ and $12 j+2$ whose projection is $m$;

$-12 j+10$ and $12 j+11$ whose projection is $3 m+1$

are determined by the already placed entries, and the exactly-1-4-adjacency constraint(see Fig. 3. $i)-i i)$ );

iii) columns $12 j+8$ and $12 j+9$ whose projections are $2 m+1$ and $2 m$, respectively, and rows $6 i+2$ and $6 i+3$ whose projections are $2 n$ and $4 n$, respectively, are also determined by the previous placements of entries, and again by the exactly-1-4-adjacency constraint (see Fig. 3, iv)).

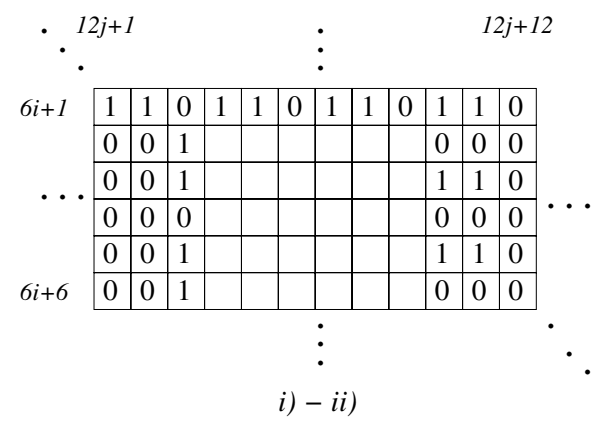

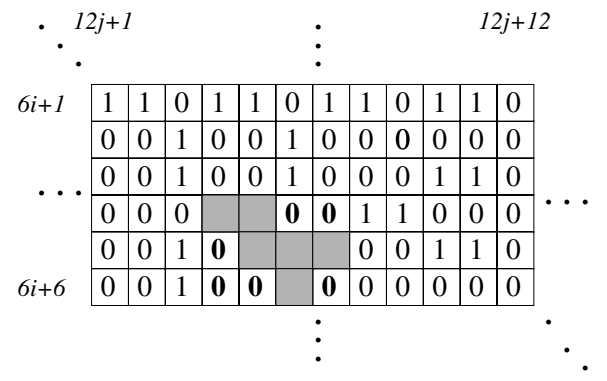

iii)

Fig. 3. The reconstruction of the fixed parts of a generic solution of instance $I^{\prime}$

Finally, the boldface elements 0 in Fig. 3 , iii) are set by the exactly-1-4adjacency constraint, and finally, the remaining grey positions are those elements of $M^{\prime}$ which are not fixed, and which may eventually differ from one solution to another. We refer to (the shape of) each of these configurations by $\mathcal{S}$. The reader can check that the found $\mathcal{S}$ is the same as that associated to each color in Fig. 2.

Now the definition of the matrix $M$ from $M^{\prime}$ is straightforward: for each $0 \leq i<m$ and $0 \leq j<n$

- if $M[6 i+4,12 j+5]^{\prime}=M[6 i+5,12 j+5]^{\prime}=1$, then $M[i+1, j+1]=c$;

- if $M[6 i+5,12 j+5]^{\prime}=M[6 i+5,12 j+6]^{\prime}=1$, then $M[i+1, j+1]=y$;

- if $M[6 i+5,12 j+6]^{\prime}=M[6 i+7,12 j+6]^{\prime}=1$, then $M[i+1, j+1]=b$;

- if $M[6 i+5,12 j+6]^{\prime}=M[6 i+5,12 j+7]^{\prime}=1$, then $M[i+1, j+1]=r$; 
The correctness of this last step deeply relies on the assumption that no other configurations except those four coding a color are possible inside each $\mathcal{S}$.

To prove it, we remind that

- each of the four colors is associated to a maximal placements of 1 s in $\mathcal{S}$;

- no other maximal placements exist. This fact can be easily checked by an exhaustive search.

So, let us consider the vectors of vertical projections of the four configurations $\mathcal{S}$ in Fig. 2 ,

$$
v_{c}=(0,2,0,0), \quad v_{y}=(0,1,1,0), \quad v_{b}=(1,1,2,0) \quad \text { and } \quad v_{r}=(1,1,1,1),
$$

and the vectors of vertical projections of the four remaining non maximal configurations of $\mathcal{S}$ shown in Fig. 4

$$
v_{1}=(1,1,0,0), \quad v_{2}=(0,0,2,0), \quad v_{3}=(0,0,1,1) \quad \text { and } \quad v_{0}=(0,0,0,0) .
$$

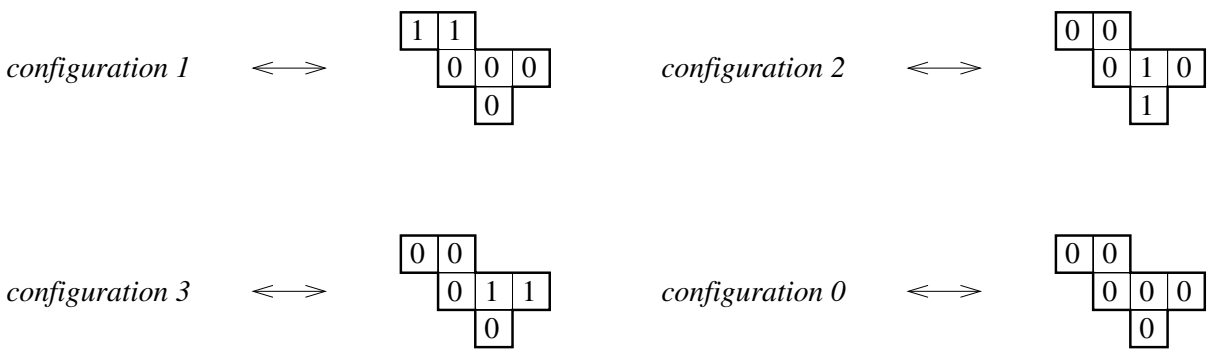

Fig. 4. The four non-maximal configurations of $\mathcal{S}$

We proceed by contradiction, and we assume that there exists a solution of $I$ which has at least one non maximal placements of elements in one of the $\mathcal{S}$ s, say $\mathcal{S}^{\prime}$. In that case no color can be associated to $\mathcal{S}^{\prime}$.

The vertical projections of the $m-1$ configurations which lie above and below $\mathcal{S}^{\prime}$, i.e. which share with it the same columns in $M^{\prime}$, together with $\mathcal{S}^{\prime}$ itself, can be expressed both as linear combination of $v_{c}, v_{y}, v_{b}$, and $v_{r}$, and as linear combination of all and eight the vectors of projections as follows:

$$
\begin{aligned}
& l 1: k_{1} v_{c}+k_{2} v_{y}+k_{3} v_{b}+k_{4} v_{r}, \\
& l 2: k_{1}^{\prime} v_{c}+k_{2}^{\prime} v_{y}+k_{3}^{\prime} v_{b}+k_{4}^{\prime} v_{r}+k_{5}^{\prime} v_{1}+k_{6}^{\prime} v_{2}+k_{7}^{\prime} v_{3}+k_{8}^{\prime} v_{0} .
\end{aligned}
$$

Since the number of the involved $\mathcal{S}$ in both the linear combinations has to be fixed, and since it holds that 
- the sums of the elements of $v_{b}$ and $v_{r}$ is equal to 4 , and it is greater than the sums of each of six remaining vectors;

- the sum of the elements of $v_{0}$ is zero, which is the minimum of the sums of each of the other vectors;

- the sums of the elements of each of the remaining vectors are 2 ,

then it follows that $k_{3}+k_{4}=k_{3}^{\prime}+k_{4}^{\prime}$.

Since the contribution to the first entry in both the linear combinations is given only by $v_{b}, v_{r}$ and $v_{1}$, it also holds that $k_{5}^{\prime}=0$.

On the other hand, the contribution to the second entry in both the linear combinations, decreased by $k_{3}+k_{4}$, is now restricted only to the vectors $v_{c}$ and $v_{y}$, so we have $k_{1}+k_{2}=k_{1}^{\prime}+k_{2}^{\prime}$.

As a consequence, we reach $k_{6}^{\prime}=k_{7}^{\prime}=k_{8}^{\prime}=0$, against the assumption that there exists in $l 2$ at least one vector of projections different from the maximal ones.

Now we can state the following

Theorem 1. The problem Consistency $\left(\mathcal{N}_{4}^{=1}, H, V\right)$ is NP-complete.

The proof is a direct consequence of the defined reduction, and, as a consequence, we have

Corollary 1. The problem Reconstruction $\left(\mathcal{N}_{4}^{=1}, H, V\right)$ is NP-hard.

The following example tries to clarify the reduction

Example 1. Let us consider the instance $I=(H 3, V 3)$ where

$$
H 3=((0,1,0),(1,0,1),(0,1,1)) \text { and } V 3=((0,2,1),(0,1,0)) .
$$

\begin{tabular}{|c|c|c|c|c|c|c|c|c|c|c|c|c|c|c|c|c|c|c|c|c|c|c|c|c|c|c|}
\hline 1 & 1 & 0 & 1 & 1 & 0 & 1 & 1 & $\begin{array}{ll}0 \\
\end{array}$ & 1 & 1 & \begin{tabular}{l|l}
0 \\
\end{tabular} & 1 & 1 & 0 & \begin{tabular}{l|l|}
1 \\
\end{tabular} & 1 & 0 & 1 & 1 & 0 & 1 & 1 & & & & \\
\hline 0 & 0 & 1 & 0 & 0 & 1 & 0 & 0 & 0 & 0 & 0 & 0 & 0 & 0 & 1 & 0 & 0 & 1 & 0 & 0 & 0 & 0 & 0 & 0 & & & \\
\hline 0 & 0 & 1 & 0 & 0 & 1 & 0 & 0 & 0 & 1 & 1 & \begin{tabular}{l|l}
0 \\
\end{tabular} & 0 & 0 & 1 & 0 & 0 & 1 & 0 & 0 & 0 & 1 & 1 & 0 & & & \\
\hline 0 & 0 & 0 & 1 & 1 & 0 & 0 & 1 & 1 & 0 & 0 & 0 & 0 & 0 & 0 & 0 & 1 & 0 & 0 & 1 & 1 & 0 & 0 & 0 & & & \\
\hline 0 & 0 & 1 & 0 & 0 & 1 & 0 & 0 & 0 & 1 & 1 & 0 & 0 & 0 & 1 & 0 & 1 & 0 & 0 & 0 & 0 & 1 & 1 & 0 & & & \\
\hline 0 & 0 & 1 & 0 & 0 & 1 & 0 & 0 & 0 & 0 & 0 & 0 & 0 & 0 & 1 & 0 & 0 & 0 & 0 & 0 & 0 & 0 & 0 & 0 & & & \\
\hline 1 & 1 & 0 & 1 & 1 & 0 & 1 & 1 & 0 & 1 & 1 & 0 & 1 & 1 & 0 & \begin{tabular}{l|l|l}
1 &
\end{tabular} & 1 & 0 & 1 & 1 & 0 & 1 & 1 & 0 & & & \\
\hline 0 & 0 & 1 & 0 & 0 & 1 & 0 & 0 & 0 & 0 & 0 & 0 & 0 & 0 & 1 & 0 & 0 & 1 & 0 & 0 & 0 & 0 & 0 & 0 & & & \\
\hline 0 & 0 & 1 & 0 & 0 & 1 & 0 & 0 & 0 & 1 & 1 & 0 & 0 & 0 & 1 & 0 & 0 & 1 & 0 & 0 & 0 & 1 & 1 & \begin{tabular}{|l|l|} 
\\
\end{tabular} & & & \\
\hline 0 & 0 & 0 & 1 & 1 & 0 & 0 & 1 & 1 & 0 & 0 & \begin{tabular}{ll|} 
\\
\end{tabular} & 0 & 0 & 0 & 0 & 0 & 0 & 0 & 1 & 1 & 0 & 0 & 0 & & & $y$ \\
\hline 0 & 0 & 1 & 0 & 0 & 1 & 1 & 0 & 0 & 1 & 1 & 0 & 0 & 0 & 1 & 0 & 1 & 1 & 0 & 0 & 0 & 1 & 1 & \begin{tabular}{|l|l|} 
\\
\end{tabular} & & & $r$ \\
\hline 0 & 0 & 1 & 0 & 0 & 0 & 0 & 0 & 0 & 0 & 0 & 0 & 0 & 0 & 1 & 0 & 0 & 0 & 0 & 0 & 0 & 0 & 0 & \begin{tabular}{|l|} 
\\
\end{tabular} & & & \\
\hline 1 & 1 & 0 & 1 & 1 & 0 & 1 & 1 & 0 & 1 & 1 & 0 & 1 & 1 & 0 & 1 & 1 & 0 & 1 & 1 & 0 & 1 & 1 & \begin{tabular}{|l|} 
\\
\end{tabular} & & & \\
\hline 0 & 0 & 1 & 0 & 0 & $\begin{array}{ll}1 \\
\end{array}$ & 0 & 0 & 0 & 0 & 0 & 0 & 0 & 0 & 1 & 0 & 0 & 1 & 0 & 0 & 0 & 0 & 0 & \begin{tabular}{|l|} 
\\
\end{tabular} & & & \\
\hline 0 & 0 & 1 & 0 & 0 & 1 & \begin{tabular}{l|l}
0 \\
\end{tabular} & 0 & 0 & 1 & 1 & 0 & 0 & 0 & 1 & \begin{tabular}{l|l}
0 \\
\end{tabular} & 0 & 1 & 0 & 0 & 0 & 1 & 1 & 0 & & & \\
\hline 0 & 0 & 0 & 1 & 1 & 0 & 0 & 1 & 1 & 0 & 0 & 0 & 0 & 0 & 0 & 1 & 1 & 0 & 0 & 1 & 1 & 0 & 0 & 0 & & & \\
\hline 0 & 0 & 1 & 0 & 0 & 1 & 0 & 0 & 0 & 1 & 1 & 0 & 0 & 0 & 1 & 0 & 0 & 1 & 1 & 0 & 0 & 1 & 1 & 0 & & & \\
\hline 0 & 0 & 1 & 0 & 0 & 1 & 0 & 0 & 0 & 0 & 0 & 0 & 0 & 0 & 1 & 0 & 0 & 0 & 0 & 0 & 0 & 0 & 0 & 0 & & & \\
\hline 1 & & 0 & 1 & 1 & 0 & 1 & 1 & 0 & 1 & 1 & 0 & 1 & 1 & 0 & 1 & 1 & 0 & 1 & 1 & 0 & 1 & 1 & 0 & & & \\
\hline
\end{tabular}

Fig. 5. The correspondence between an instance of 3-color and a matrix in $\mathcal{N}_{4}^{=1}$ 
for which the solutions are 3 -color matrices of dimension $3 \times 2$.

Following what defined in paragraph 3, the correspondent instance $I^{\prime}=$ $(H, V)$ of Consistency $\left(\mathcal{N}_{4}^{=1}, H, V\right)$ is

$$
\begin{aligned}
& H=(16,4,8,7,8,3,16,4,8,6,10,2,16,4,8,8,9,3,16) \text { and } \\
& V=(4,4,12,7,7,11,5,7,3,10,10,0,4,4,12,5,8,8,5,7,3,10,10,0) .
\end{aligned}
$$

Figure 5 depicts a binary matrix $M^{\prime}$ which has $H$ and $V$ as vectors of projections, together with its associated 3-color matrix, having projections $H 3$ and $V 3$. Again we underline the property that there is no way of obtaining a matrix $M^{\prime}$ whose configurations of 0 s and 1 s inside the grey zone are not in correspondence with a color.

\section{Conclusion}

In this paper we have studied the computational complexity of the consistency problem for the class $\mathcal{N}_{4}^{=1}$. This is one the simplest examples of a class of binary matrices satisfying adjacency constraints. However, our studies in that direction are just at the beginning, and a systematical analysis of all the other cases of 4-adjacency constraints and their extension to a general framework where the adjacent constraint involve different sets of neighborhood elements are work in progress. Our attention is also attracted by the presence in $\mathcal{N}_{4}^{=1}$ of some subclasses which allow a fast reconstruction strategy, and which show some interesting geometrical aspects.

\section{References}

1. Brunetti, S., Costa, M.C., Frosini, A., Jarray, F., Picouleau, C.: Reconstruction of binary matrics under adjacency constraints. In: Herman, G.T., Kuba, A. (eds.) Advances in Discrete Tomography and its Applications, pp. 125-150. Birkhäuser, Basel (2007)

2. Chrobak, M., Couperus, P., Dürr, C., Woeginger, G.: A note on tiling under tomographic constraints. Theor. Comp. Sc. 290, 2125-2136 (2003)

3. Chrobak, M., Dürr, C.: Reconstructing Polyatomic Structures from X-Rays: NP Completness Proof for three Atoms. Theor. Comp. Sc. 259, 81-98 (2001)

4. Herman, G., Kuba, A.: Discrete Tomography: Foundations, Algorithms and Applications. Birkhauser, Basel (1999)

5. Herman, G., Kuba, A.: Advances in Discrete Tomography and its Applications. Birkhäuser, Basel (2007)

6. Jarray, F.: Résolution de problèmes de tomographie discrète. Applications à la planification de personnel, Ph. D. thesis, CNAM, Paris, France (2004)

7. Ryser, H.J.: Combinatorial properties of matrices of zeros and ones. Canad. J. Math. 9, 371-377 (1957)

8. Ryser, H.J.: Combinatorial mathematics, Mathematical Association of America and Quinn \& Boden, Rahway, New Jersey (1963) 